\title{
Manual Testing of Respiratory Muscles in Adults with Asthma: A Intra and Interrater Reliability and Validity Study
}

Yannely Serrano-Villar ( $\sim$ yannely.serrano.villar@gmail.com )

Universidad Industrial de Santander

Eliana-Isabel Rodríguez-Grande

Universidad del Rosario

María Solange Patiño Segura

Universidad Industrial de Santander

\section{Research Article}

Keywords: asthma, Manual Muscle Testing, reliability, validity, respiratory muscles, muscle strength

Posted Date: January 6th, 2022

DOI: https://doi.org/10.21203/rs.3.rs-1174478/v1

License: (c) (i) This work is licensed under a Creative Commons Attribution 4.0 International License.

Read Full License 


\section{Abstract}

Background: Manual Muscle Testing (MMT) is a useful tool to evaluate ventilatory mechanics in adults with asthma. However, in the reviewed literature, there are few studies that report psychometric features of this test. Therefore, the present study aimed to evaluate MMT reliability and validity in respiratory muscles in adults with asthma.

Methods: It was a cross-sectional study. Muscle strength (MMT and static respiratory pressures), sociodemographic and anthropometric variables related to the disease were evaluated. Measurements were carried out by two independent evaluators The reliability of MMT was analyzed with the weighted kappa and the convergent validity was evaluated by comparing the MTT and the respiratory pressure measurements using Pearson's correlation coefficient. The level of significance was $p<0.05$.

Results: Twenty-six adults with stable asthma participated in the study. The intrarater reliability for MMT was between moderate and substantial (kappa=0.45-0.88) for all evaluated muscles while the interrater reliability was slight and fair for intercostal muscles (kappa=0.07-0.24), and fair and substantial (kappa $=0.36-0.75)$ for other muscles. The convergent validity of MMT and respiratory pressures was low $(r=0.20-0.48)$.

Conclusions: MMT is a reliable measurement that can be used to evaluate respiratory muscle strength in adults with asthma. This study support MMT application for respiratory muscles at clinical settings when more objective measures such as MIP and MEP are not available.

Considering that the MMT is a useful, practical, low-cost tool commonly used by physiotherapists, future studies could evaluate the convergent validity compared with dynamometry or electromyography of the respiratory muscles.

\section{Background}

Worldwide, asthma is considered the most common chronic respiratory disease. It affects as many as 334 million people reaching the 14th place among the most important diseases in terms of extent and time period of disability. ${ }^{1}$ Asthma is characterized by chronic inflammation of the airways accompanied of airflow obstruction; ${ }^{2}$ causing an intermittent load on respiratory muscles and muscle overload during acute exacerbations ${ }^{3}$. The resulting muscle dysfunction is evidenced in the decrease of the muscle strength, ${ }^{4}$ and in the reduced net excitation of inspiratory motor neurons. ${ }^{3}$

To know the respiratory muscle function state is valuable to determine functional consequences and level of asthma control. Consequently, several technological strategies have been developed for its assessment, namely, measurement of maximal inspiratory and maximal expiratory pressures (MIP and MEP), ${ }^{5}$ spirometry ${ }^{6}$ and electromyography. ${ }^{7}$ Nonetheless, since these procedures are not available in all clinical settings, monitoring of breathing pattern, costal mobilization, and assessment of muscle contraction with palpation ${ }^{5}$ are traditionally used as part of the Manual Muscle Testing (MMT). The latter 
has been recognized as a simple, economical and accessible tool. ${ }^{8}$ In spite of the advantages, the MMT psychometric features have only been evaluated on limb musculature as the reviewed literature shows. ${ }^{9}$

The availability of reliable and valid measuring tools within the clinical ambit, allows to make an unbiased assessment, suggest a diagnosis, and implement treatments oriented to improve ventilatory mechanics compromised by the asthma. ${ }^{3}$ Additionally, for research, it can be useful to support outcomes derived from physiotherapeutic interventions by reducing the likelihood that results be affected by measurement variability.

According to the aforementioned, it is necessary to assess the psychometric properties of tools used in clinical practice and compare them with tests and measurements of proven validity and reliability. In that sense, MMT reliability, and validity against MIP and MEP should be evaluated. In this way, ventilatory function assessment of patients with asthma can be complemented for monitoring disease control, providing support to therapeutical programs to control symptoms, preventing complications, and improving function and life quality of this population. ${ }^{2}$ Therefore, the research question of present study were: What is the intra and interrater reliability of MMT of the diaphragm, external intercostal and abdominal muscles in a population of patients with asthma?, and What is the convergent validity of MTT against MIP and MEP in a population of patients with asthma?.

\section{Methods}

The reliability and validity evaluation of the diagnostic tests were carried out using a cross-sectional sampling. ${ }^{10}$

Subjects: The study group included adults with asthma in stable phase of disease, according to the criteria proposed by the GINA, which include the control of signs and symptoms, and the result of functional tests. ${ }^{2}$ Patients were excluded if comorbidities such as heart diseases, uncontrolled arterial hypertension, recent lung biopsy, spinal cord injury, ocular lesion, tracheotomy, surgery or trauma of upper airways, hemodynamic instability, pregnancy, respiratory infections ${ }^{11}$ were present. Also, in case of sequelae of musculoskeletal or neurologic disorders compromising thoracic mobility and muscle control; ${ }^{12}$ or a lack of voluntary effort during spirometry, defined by a Peak Expiratory Flow (PEF) or by a Forced Expiratory Flow at $25 \%\left(\mathrm{FEF}_{25 \%}\right)$ under $60 \%$ of the predicted value (13). Measurements were taken in the Laboratory of Movement Analysis, School of Physiotherapy, Universidad Industrial de Santander.

Evaluators: Two clinical-experienced physiotherapists participated in the study. They standardized verbal commands and hand contacts and were trained in the application of tests, in order to avoid classification bias.

\section{Procedures:}


The protocol comprised measurements that should be done in three days ( 2 and 8 days apart). On day 0 screening and familiarization were carried out; anthropometric, spirometric, sociodemographic, diseaserelated and monitoring-related variables were measured, and familiarization with muscle strength variables were attained. On the second day, MMT was measured independently by two evaluators. On the third day, MMT was measured again by the two evaluators; additionally, one of the evaluators always measured the Maximal Inspiratory and Expiratory Pressures (MIP and MEP). All variables were measured at a random order. Vital signs were monitored by one of the evaluators at the beginning and end of each session. Both evaluators were blinded for the previous measurements and for those from each other. Additionally, evaluations were done at the same time of day. Patients were requested to continue the medical treatment during the study (Figure 1).

\section{Measurements}

Spirometry. A spirometer (Spirobank G., MIR SRL) was used following technical procedures recommended by the American Thoracic Society and the European Respiratory Society. ${ }^{6,13}$ Subjects completed at least three maximal forced expiratory maneuvers and $\mathrm{PEF}$ and $\mathrm{FEF}_{25 \%}$ were recorded; if these variables fell below $60 \%$ of the predicted value ${ }^{6},{ }^{13}$ the participant was excluded from the study. The forced expiratory volume during the first second $\left(F^{\prime} V_{1}\right)$, the forced vital capacity $(F V C)$ and the $F E V_{1} / F V C$ ratio were also recorded.

Anthropometric variables. Corporal weight (Kg) was measured using a portable, digital, and calibrated scale; size $(\mathrm{m})$ was measured with an inextensible metric tape of $1 \mathrm{~mm}$ precision; and the Body Mass Index (BMI=weight/size ${ }^{2}$ ) was calculated. Recommendations from the Anthropometry Procedures Manual of the National Health and Nutrition Examination Survey, elaborated by the Centers for Disease Control and Prevention, ${ }^{14}$ were followed.

Monitoring variables. Heart rate (beats/min), rate of breathing (respirations/min), arterial blood pressure $(\mathrm{mm} \mathrm{Hg})$, and oxygen saturation $\left(\mathrm{SaO}_{2}\right.$; percentage) with $2 \%$ precision were recorded. Additionally, lung auscultation findings, breathing difficulty assessed through the Borg Scale, and signs of respiratory distress, were also recorded. ${ }^{15,16}$

Variables of muscle strength. Muscle strength was assessed by MMT and static respiratory pressures measurement. MMT was based on palpation, thoracic mobility observation, and manual resistance applied against a contracting muscle, in accordance with the ordinal grading system from the Medical Research Council (MRC). ${ }^{17}$ The protocol implemented for this study took into account evaluator and subject postures, the applied hand contacts, the muscle palpation pressure, and the verbal command emitted. The grading scale was different for the inspiratory and the expiratory musculatures. A "normal /good /fair /poor /trace /null" scale was used for diaphragm and external intercostal muscles, taking into account the thoracic expansion, expulsion of the hand, and the respiratory function. The terms functional, slightly functional, non-functional and null were used for abdominal muscles, according to the characteristics of the coughing mechanism. ${ }^{8,18}$ The evaluator began the test by emitting the 
corresponding verbal command while observing the muscle contraction; then placed his/her hands on the indicated muscle points for palpating its contraction without resistance; and finally, applied manual resistance against the muscle movement. ${ }^{8,18}$ Tests for every muscle were made twice with 1-min interval between each effort, and the higher value was recorded. Images of diaphragm, external intercostal, and abdominal muscles evaluation are shown in Figures 2, 3, and 4 respectively.

MIP and MEP measurements were done using a manometer with nozzle and valves for censing pressure changes. MIP is considered the maximal subatmospheric pressure generated against an occluded airway from residual volume (Müller maneuver), sustained for one second. MEP was measured from the total lung capacity (TLC) and exerting the maximal expiratory pressure against the occluded airway (Valsalva maneuver) for one second. ${ }^{19}$

Statistics. A25-35 individual-sample was calculated to be suitable for determining MMT psychometric features after considering the use of two replicates and assuming $80 \%$ power, a significance level of $5 \%$ and a loss of $20 \% .^{20}$

Measures of central tendency and dispersion were calculated to characterize the population according to nature and distribution of variables. Both, intrarater and interrater reliability of MMT were evaluated using weighted kappa. ${ }^{10}$ Landis and Koch guidelines ${ }^{21}$ for interpreting kappa values, were implemented as follows, slight (kappa=0.0-0.2), fair (kappa=0.21-0.40), moderate (kappa=0.41-0.60), substantial (kappa=0.61-0.80), and almost perfect (kappa=0.8-1.00) agreement. Convergent validity of MTT and static pressures was established by Pearson correlation coefficient; the expected level of correlation was classified in accordance with data from Carter et al. ${ }^{22}$ as follows, small $(r=0.0-0.25)$, low $(r=0.26-0.49)$, moderate $(r=0.50-0.69)$, high $(r=0.70-0.89)$, and very high $(r=0.90-1.00)$.

\section{Results}

Flow of participants. Twenty-nine adults with stable asthma were enrolled for the study; three did not complete the programmed evaluations; therefore, only 26 patients were included in the study (Figure 1); 16/26 (61.54\%) were female. Table 1 describes general, disease-related and spirometric characteristics of the patients. Spirometric pattern was normal in all participants as evidenced by $\mathrm{FEV}_{1}$ and $\mathrm{FVC}$, and $\mathrm{FEV}_{1} / \mathrm{FVC}$ that were respectively above $80 \%$ and $70 \%$ of the predicted values. ${ }^{6}$ 
Table 1

Characteristics of sample $(\mathrm{N}=26)$

\begin{tabular}{|c|c|}
\hline Variable & Data \\
\hline Age, years (percentile 25;75) & $24.5(20 ; 32)$ \\
\hline Weight, kg (SD) a & $69.4(16.11)$ \\
\hline Size, m (SD) & $1.6(0.08)$ \\
\hline $\mathrm{BMI}^{\mathrm{b}}, \mathrm{Kg} / \mathrm{m}^{2}(\mathrm{SD})$ & $25.5(5.24)$ \\
\hline Schooling, approved years (SD) & $14.6(3.43)$ \\
\hline \multicolumn{2}{|l|}{ Asthma Features } \\
\hline Time of disease evolution, years (SD) & $18.1(9.31)$ \\
\hline Time after last acute exacerbation, months (percentile $25 ; 75$ ) & $7(4 ; 36)$ \\
\hline \multicolumn{2}{|l|}{ Spirometric values } \\
\hline $\mathrm{PEF}^{\mathrm{c}}(\mathrm{SD})$ & $93.3(20.88)$ \\
\hline $\mathrm{FEF}_{25 \%}{ }^{\mathrm{d}}(\mathrm{SD})$ & $74.1(23.66)$ \\
\hline $\mathrm{FEV}_{1}^{\mathrm{e}}(\mathrm{SD})$ & $82.8(13.82)$ \\
\hline $\mathrm{FVC}^{f}(\mathrm{SD})$ & $91.2(9.79)$ \\
\hline $\mathrm{FEV}_{1} / \mathrm{FVC}(\mathrm{SD})$ & $89.8(10.92)$ \\
\hline
\end{tabular}

a: Standard Deviation; b: Body Mass Index; c: Peak Expiratory Flow; d: Forced Expiratory Flow at 25\% of FVC; e: Forced Expiratory Volume during the first second; f: Forced Vital Capacity

Reliability of MMT. Intrarater reliability was similar in both evaluators; the agreement was substantial (kappa $=0.78-0.88)$ for external superior intercostal and abdominal muscles, and moderate (kappa $=0.45-$ 0.59) for anterior and posterior diaphragm. Confidence intervals were wide for all musculature evaluated. The intrarater reliability findings are shown in Table 2. 
Table 2

MMT Intrarater Reliability ( $\mathrm{N}=26)$

\begin{tabular}{|c|c|c|c|c|}
\hline Muscle & Agreement (\%) & Expected agreement (\%) & Kappa & $95 \% \mathrm{Cl}^{\mathrm{a}}$ Normal \\
\hline \multicolumn{5}{|l|}{ Evaluator 1} \\
\hline Anterior diaphragm & 90.38 & 76.18 & 0.59 & $0.26-0.925$ \\
\hline Lateral diaphragm & 95.19 & 76.70 & 0.79 & $0.57-1.00$ \\
\hline Posterior diaphragm & 92.31 & 81.36 & 0.58 & $0.28-0.89$ \\
\hline Superior $\mathrm{ICs}^{\mathrm{b}}$ & 96.15 & 66.57 & 0.88 & $0.61-1.00$ \\
\hline Inferior ICs ${ }^{b}$ & 76.92 & 50.30 & 0.53 & $0.21-0.85$ \\
\hline Rectus abdominis & 92.31 & 64.20 & 0.78 & $0.46-1.00$ \\
\hline Oblique ones & 92.31 & 64.20 & 0.78 & $0.46-1.00$ \\
\hline \multicolumn{5}{|l|}{ Evaluator 2} \\
\hline Anterior diaphragm & 93.16 & 87.44 & 0.45 & $0.08-0.83$ \\
\hline Lateral diaphragm & 94.87 & 88.76 & 0.54 & $0.18-0.90$ \\
\hline Posterior diaphragm & 91.35 & 80.10 & 0.56 & $0.27-0.85$ \\
\hline Superior $I C s^{b}$ & 98.08 & 89.64 & 0.81 & $0.50-1.00$ \\
\hline Inferior ICs ${ }^{b}$ & 96.15 & 90.98 & 0.57 & $0.12-1.00$ \\
\hline Rectus abdominis & 96.15 & 82.54 & 0.77 & $0.22-1.00$ \\
\hline Oblique ones & 96.15 & 82.54 & 0.77 & $0.22-1.00$ \\
\hline
\end{tabular}

The interrater reliability was between slight and substantial during the second and third evaluation days. Confidence intervals were wide, but reduced for lateral diaphragm. The findings of interrater reliability for each muscle is shown in Table 3. 
Table 3

MMT Interrater Reliability of ( $\mathrm{N}=26)$

\begin{tabular}{|c|c|c|c|c|}
\hline Muscle & Agreement (\%) & Expected agreement (\%) & Kappa & $95 \% \mathrm{Cl}^{\text {a }}$ Normal \\
\hline \multicolumn{5}{|l|}{ Measurement 1} \\
\hline Anterior diaphragm & 94.87 & 87.57 & 0.58 & $0.28-0.88$ \\
\hline Lateral diaphragm & 94.23 & 76.63 & 0.75 & $0.53-0.97$ \\
\hline Posterior diaphragm & 88.46 & 78.11 & 0.47 & $0.09-0.84$ \\
\hline Superior ICs ${ }^{b}$ & 82.69 & 80.92 & 0.09 & $-0.05-0.24$ \\
\hline Inferior ICs ${ }^{b}$ & 86.54 & 84.91 & 0.10 & $-0.17-0.39$ \\
\hline Rectus abdominis & 80.77 & 69.53 & 0.36 & $-0.03-0.76$ \\
\hline Oblique ones & 80.77 & 69.53 & 0.36 & $-0.03-0.76$ \\
\hline \multicolumn{5}{|l|}{ Measurement 2} \\
\hline Anterior diaphragm & 94.23 & 77.07 & 0.74 & $0.48-1.00$ \\
\hline Lateral diaphragm & 97.01 & 88.79 & 0.73 & $0.49-0.97$ \\
\hline Posterior diaphragm & 93.27 & 83.80 & 0.58 & $0.25-0.91$ \\
\hline Superior ICs ${ }^{b}$ & 81.73 & 80.25 & 0.07 & $-0.05-0.20$ \\
\hline Inferior ICs ${ }^{b}$ & 88.46 & 84.76 & 0.24 & $0.03-0.45$ \\
\hline Straight abdominal & 92.31 & 73.67 & 0.70 & $0.26-1.00$ \\
\hline Oblique ones & 92.31 & 73.67 & 0.70 & $0.26-1.00$ \\
\hline
\end{tabular}

Additionally, the overall interrater reliability was analyzed considering both assessments results as well as the overall reliability between the two evaluation days (Table 4). The interrater reliability was substantial for diaphragm, moderate for abdominal and slight for intercostal muscles; while the intermeasurement reliability was substantial for all muscle groups. 
Table 4

Overall Reliability $(\mathrm{N}=26)$

\begin{tabular}{|lllll|}
\hline Muscle & Agreement (\%) & $\begin{array}{l}\text { Expected } \\
\text { agreement (\%) }\end{array}$ & Kappa & 95\% Cl Normal \\
\hline Interrater reliability & & & \\
\hline Diaphragm & 95.19 & 80.10 & 0.75 & $0.49-1.00$ \\
\hline Intercostal ones & 42.31 & 33.43 & 0.13 & $-0.01-0.28$ \\
\hline Abdominal ones & 80.77 & 66.42 & 0.42 & $0.06-0.79$ \\
\hline Inter-measurement reliability & & & \\
\hline Diaphragm & 95.19 & 79.36 & 0.76 & $0.54-0.98$ \\
\hline Intercostal ones & 88.46 & 50.00 & 0.76 & $0.51-1.00$ \\
\hline Abdominal ones & 92.31 & 64.20 & 0.78 & $0.46-1.00$ \\
\hline
\end{tabular}

Convergent validity of MMT and static respiratory pressures. Overall, correlation between static respiratory pressures and MMT was between small and low for both, inspiratory and expiratory muscles (Table 5).

Table 5

Correlation between static respiratory pressures and $\operatorname{MMT}(\mathrm{N}=26)$

\begin{tabular}{|lrl|}
\hline Muscle group & $\mathbf{R}$ & $\mathbf{9 5 \%} \mathrm{Cl}$ \\
\hline \multicolumn{2}{|l|}{ Inspiratory (absolute average) } & \\
\hline MIP and diaphram & 0.25 & $-0.15-0.58$ \\
\hline MIP and external intercostal & 0.48 & $0.12-0.73$ \\
\hline MIP and inspiratory & 0.39 & $0.01-0.67$ \\
\hline Expiratory (absolute average) & & \\
\hline MEP and abdominal & 0.20 & $-0.19-0.55$ \\
\hline r= Pearson's correlation coefficient & \\
\hline
\end{tabular}

\section{Discussion}

MMT intrarater reliability, was substantial for diaphragm, external intercostal and abdominal muscles (Table 4). These results could be attributed to standardization of the protocol. Unfortunately, it was not possible to compare the observed results with other studies since MMT reliability in respiratory muscles has not been previously assessed. ${ }^{9,23,24}$ 
The intrarater reliability level was different when analyzing specifically each muscle group according to subgroups; it varied from almost perfect to moderate. These variations cannot be attributed to changes in muscle strength since the elapsed time between measurements is not enough to show significant changes in muscle strength. Such variations can be explained by analyzing biomechanics of respiratory muscles. Then, the almost perfect agreement for superior external intercostal muscles and the substantial result for abdominal muscles, can be explained owing that palpation of these muscles is a simple task due to their superficial anatomical disposition. ${ }^{18,25,26}$

Moderate to substantial reliability for diaphragm should be analyzed taking into account its function and anatomical disposition. When the diaphragm contracts, its central tendon moves downwards and the dome tends to flatten. ${ }^{18,26,27}$ Such behavior makes diaphragm palpation a complex task during the test. Additionally, the pathologically flattened diaphragm, resultant from chronic respiratory diseases, pulls the ribs laterally instead of up and outwards, decreasing the capacity to enlarge thorax dimensions. This difference in diaphragm function can hinder participant's performance during evaluation becoming an uncontrolled issue for the evaluator. ${ }^{25,28}$ On the other hand, the lower reliability observed in the inferior external intercostal muscles can be related to diaphragm interference. Diaphragmatic action depends on the union to the six lower ribs and the zone of apposition, an area where the muscle juxtaposes to the inner surface of the costal margin. ${ }^{28}$

The overall interrater reliability is influenced by the evaluators' training and standardization of the measurement protocol. Although these factors were controlled in the present study, the evaluated construct (muscle strength) by MMT involves a subjective assessment. Consequently, the variability in the observations can be attributed to differences in the resistance applied to the muscle and the grade given to the perceived performance.

Despite hand contact standardization, manual resistance was not quantified; therefore, it could differ between evaluators. These differences may affect the external intercostal muscles since the forces exerted on the ribs are transmitted to the rib cage through the joints. ${ }^{25,29,30}$ On the other side, the fair interrater reliability observed on the first day in abdominal muscles can be attributed to the lack of differentiation between the two levels of evaluation ("functional" and "weakly functional") in the grading scale used. This could cause a difference in the criteria of evaluation between both raters.

When comparing measurements of the two days, interrater reliability was greater in the second measurement for the majority of muscles (Table 3). This can be attributed to the effect of participants' test evaluation learning. In this context, Lavietes ${ }^{31}$ described a training-derived outcome when repeated measurements of MIP were done in adults with acute asthma. Possibly the same factor is present in MMT since both methods assess respiratory muscle strength. Therefore, in every new measurement, muscle performance improves as a result of previous experience.

The confidence intervals of kappa coefficients calculated for both days were wide, reflecting the typical variability of the construct evaluated and the MMT subjectivity on respiratory muscles. Variability of 
measurements can be explained by features of these groups of muscles; namely, localization within the thoracic cavity, ${ }^{18,25,27}$ and influences that costal and pulmonary biomechanics may have over them. ${ }^{12}$ Strength of muscle contraction is determined by muscle length-tension, force-velocity, and stimulating strength-frequency relationships; as well as by the contractile apparatus integrity. ${ }^{27}$ Therefore, small changes in the aforementioned factors would result in variations of muscle strength perceived through palpation.

Reliability variations in the diaphragm fibers data could be influenced by the extent of the abdominal muscles relaxation, posture at the moment of testing, and modifications in ventilatory mechanics. $8,18,25$ For external intercostal muscles, the test is based on the horizontalization and verticalization extent of the ribs, changes in intercostal spaces, enlargement of the chondrosternal angle during the breathing cycle, and the diaphragm relaxation; any change in these conditions increases measurement variability. Additionally, abdominal muscles are evaluated considering their capacity to generate expiratory flows and their localization in the thoracic wall. $8,18,32$

In addition to the variability of the measurements due to physiological characteristics, it is important to mention the sample size. Although the calculated sample size was reached, perhaps it was not enough to have a greater accuracy in the reliability measurements.

The convergent validity of MMT and the static respiratory pressures was low. The correlation analysis was done between MIP and the average score of diaphragm and external intercostal muscles; as well as between MEP and the average score of abdominal muscles. The analysis was not done specifically for each subgroup of muscles because respiratory pressures do not differentiate the strength per muscle group. ${ }^{19}$ Therefore and for the reasons that will be explained below, the MIP and MEP may not be the best comparison standard.

The low overall correlation should be analyzed considering the measurement protocol of both variables. Patients for MMT are in supine position while for measuring pressures are seated. Posture has an effect on performance of respiratory muscles, mainly, in the inspiratory ones working against resistance to surpass elasticity of the rib cage, elastic resistance of lungs, gravity force, and abdominal content. ${ }^{18,27}$

Recruitment of respiratory muscles depends on breathing type, posture and thoracic wall features ${ }^{18,27}$. Particularly, the work of diaphragm and intercostal muscles can be identified in different positions. In vertical position, during an inhalation, the diaphragm pulls phrenic center down, causing an increment of the thoracic cavity vertical. During exhalation, diaphragm relaxes, its dome lifts and the thoracic volume is reduced. In decubitus position, used for MMT, the diaphragm continues working according to the pressures it receives; specifically, in lateral decubitus position, the hemidiaphragm at the lying side is pushed by the intra-abdominal pressure, and has a more expiratory role than in other positions ${ }^{29}$. Intercostal muscles work is influenced by the ribs posture, since the strength the muscles produce is transmitted through joints and cartilage to other bones. ${ }^{18,25,27}$ 
The expiratory action of abdominal muscles can be explained by the trunk position, as its flexion facilitates the egress of air ${ }^{18}$ and by muscle activation specificity, since at the end-inspiration and onsetexpiration, the more activated (minor oblique, transversus abdominis, lateral fibers of major oblique) muscles are the ones participating less at the trunk flexion. ${ }^{18}$ Additionally, diaphragm inspiratory action at the standing and sitting postures is complemented by abdominal muscles tension facilitating an increase of abdominal pressure and avoiding abdominal protrusion during inspiration. ${ }^{25}$

Another difference between the maneuvers for testing the respiratory pressures and MMT was the resistance applied. The manometer used for evaluating the pressures, provides an occlusion to the air flow that imposes a fixed resistance to muscle work ${ }^{19}$ while in MMT, the evaluator manually imposes a resistance that was not quantified. Low correlation between static respiratory pressures and MMT can also be explained by activation of muscle groups during the maneuvers. The effort measured through MIP results from joint activation of inspiratory muscles ${ }^{33}$ while MMT discriminates the effort of each muscle group. Therefore, the low correlation can show an advantage of MMT over measurement of respiratory pressures. Unfortunately, this advantage could not be corroborated in the present study because the electromyographic activity was not evaluated.

A constraint in the study was its restriction to adults with stable asthma because MMT psychometric properties were established only for such patients. Therefore, it is recommended that psychometric features be evaluated at different asthma phases and in different age groups, as well as in other pathologies. Another study limitation was that the manual resistance applied by evaluators during MMT was not quantified. Although this parameter can be measured precisely through dynamometry, the application of this measurement for respiratory muscles (mainly, the intercostal) is difficult due to their anatomical disposition. Additionally, dynamometry is restricted in the clinical practice because of shortages of equipment. Muscle strength data measured by static respiratory pressures are difficult to compare with other studies because of the use of different scales. Consequently, reporting the measured effort as percentage is advisable because it contributes to compare different results.

According to the aforementioned, it can be concluded that MMT is a reliable measurement to evaluate respiratory muscles strength of patients with asthma. This assessment can be applied by health professionals during thorax physical examination, widening the analysis of ventilatory mechanics in each case. In spite of its subjectivity, is a useful, practical, low-cost, and easy to perform tool to assess muscle groups differentiating their fibers. When correctly applied, MMT is an efficient procedure within the clinical survey of muscle work, provided that the basic conditions for efficacy of the test are fulfilled; namely, evaluator specific training, adjacent musculature relaxation, posture, adequate hand contacts, and standardized verbal commands.

\section{Conclusions}

Intra and interrater reliability for MMT was between substantial and moderate, except for interrater reliability for superior external intercostal muscles on the second day of measurements graded as slight. 
Correlations between MMT and MIP, and MEP were low.

Results from this study support MMT application for respiratory muscles at clinical settings when more objective measures such as MIP and MEP are not available. In spite of its subjectivity, is a useful, practical, low-cost, and easy to perform tool to assess muscle groups differentiating their fibers provided that the basic conditions for efficacy of the test are fulfilled; namely, evaluator specific training, adjacent musculature relaxation, posture, adequate hand contacts, and standardized verbal commands that facilitate the touch sensitivity for muscle contraction.

According to the aforementioned, it can be concluded that MMT is a reliable measurement to evaluate respiratory muscles strength of patients with asthma. Future studies could evaluate the convergent validity compared with dynamometry or electromyography of the respiratory muscles.

\section{Abbreviations}

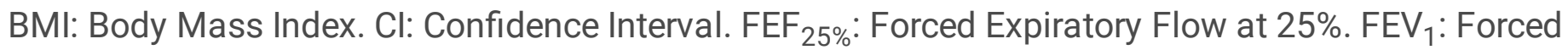
expiratory volume during the first second. FVC: Forced vital capacity. ICs: Intercostal. MEP: maximal expiratory pressure. MIP: maximal inspiratory pressure. MMT: Manual Muscle Testing. MRC: Medical Research Council. PEF: Peak Expiratory Flow. R: Pearson's correlation coefficient. SD: Standard Deviation. TLC: Total lung capacity.

\section{Declarations}

\section{Ethics approval and consent to participate}

The project was approved by the Scientific Research Ethics Committee of the School of Health of Universidad Industrial de Santander with code 7083, and it was carried out in accordance with Good Clinical Practice rules and the Ethics Principles for Medical Research Involving Human Beings that are defined in the latest revision of the Declaration of Helsinki. All of the participants voluntarily agreed to be enrolled in the study and signed an informed consent form.

\section{Consent for publication}

All of the participants voluntarily agreed to be enrolled in the study and signed an informed consent form.

\section{Availability of data and material}

Data relating to this study are avaible from the corresponding author on request.

\section{Competing interests}

The authors declare that they have no competing interests.

\section{Funding}


No funding was received.

\section{Authors' contributions}

All authors contributed to study conception and design. All authors are accountable for all aspects of the work and for the integrity of the data and the accuracy of the data analysis. All authors read and approved the final manuscript.

\section{Acknowledgements}

The study was supported by the Neurociencias y Comportamiento UIS-UPB Research Group.

\section{Author details}

${ }^{1}$ MSc in Physiotherapy, Universidad Industrial de Santander.

${ }^{2} \mathrm{MSc}$ in Physiotherapy, MS (c) in Clinical Epidemiology, Pontificia Universidad Javeriana. Teaching Professor, Universidad del Rosario, School of Medicine and Health Sciences, GI Rehabilitation Sciences: Bogotá, Cundinamarca, Colombia, PhD student in Clinical Epidemiology, Pontificia Universidad Javeriana.

${ }^{3} \mathrm{MSc}$ in Physiotherapy. Teaching Professor, Universidad Industrial de Santander, Faculty of Health, School of Physiotherapy: Bucaramanga, Santander, Colombia.

\section{References}

1. Asher I, Pearce N. Global burden of asthma among children. Int J Tuberc Lung Dis. 2014;18(11):12691278. doi:10.5588/ijtld.14.0170.

2. Bateman ED, Hurd SS, Barnes PJ, et al. Global strategy for asthma management and prevention: GINA executive summary. Eur Respir J. 2008;31(1):143-178. doi:10.1183/09031936.00138707.

3. Laghi F, Tobin MJ. Disorders of the Respiratory Muscles. Am J Respir Crit Care Med. 2003;168(1):1048. doi: $10.1164 / \mathrm{rccm} .2206020$.

4. Stell IM, Polkey MI, Rees PJ, Green M, Moxham J. Inspiratory Muscle Strength in Acute Asthma. CHEST. 2001;120(3):757.

5. Polkey MI, Green M, Moxham J. Measurement of respiratory muscle strength. Thorax. 1995;50(11):1131-1135. doi:10.1136/thx.50.11.1131.

6. Pellegrino R, Viegi G, Brusasco V, et al. Interpretative strategies for lung function tests. Eur Respir J. 2005;26(5):948-968. doi:10.1183/09031936.05.00035205.

7. ATS/ERS Statement on Respiratory Muscle Testing. Am J Respir Crit Care Med. 2002;166(4):518-624. doi:10.1164/rccm.166.4.518. 
8. Hislop H, Montgomery J, Connolly B. Pruebas Funcionales Musculares. 4 ed. Madrid: Marbán Libros; 2002.

9. Cuthbert SC, Goodheart GJ. On the reliability and validity of manual muscle testing: a literature review. Chiropr Osteopat. 2007;15:4. doi:10.1186/1746-1340-15-4.

10. Luis Orozco. Medición En Salud. Diagnóstico Y Evaluación de Resultados. Un Manual Crítico Más Allá de Lo Básico. 1 edición. Bucaramanga: Publicaciones UIS; 2010.

11. Luis Maestu, Julia Garcia. Lung function tests in clinical decision-making. 2012. 48(5):161-169.

12. Gosselink R, Stam H. European Respiratory Monograph 31: Lung Function Testing. European Respiratory Society; 2005.

13. Miller MR, Hankinson J, Brusasco V, et al. Standardisation of spirometry. Eur Respir J. 2005;26(2):319338. doi:10.1183/09031936.05.00034805.

14. CDC. CDC Works 24/7. Centers for Disease Control and Prevention. https://www.cdc.gov/index.htm. Published June 5, 2017. Accessed June 8, 2017.

15. Pryor JA, Prasad AS. Physiotherapy for Respiratory and Cardiac Problems: Adults and Paediatrics. Elsevier Health Sciences; 2008.

16. Heyward VH. Evaluación y prescripción del ejercicio. Editorial Paidotribo; 2006.

17. James MA. Use of the Medical Research Council Muscle Strength Grading System in the Upper Extremity. J Hand Surg. 2007;32(2):154-156. doi:10.1016/j.jhsa.2006.11.008.

18. Kendall, Florence Peterson, Elizabeth Kendall McCreary. Kendall's Músculos Pruebas Funcionales, Postura Y Dolor. Madrid: Marbán Libros; 2007.

19. Cavalcante Marcelino AMF, da Silva HJ. Papel da pressão inspiratória máxima na avaliação da força muscular respiratória em asmáticos - Revisão sistemática. Rev Port Pneumol. 2010;16(3):463-470. doi:10.1016/S0873-2159(15)30042-8.

20. Kraemer HC, Kupfer DJ. Size of Treatment Effects and Their Importance to Clinical Research and Practice. Biol Psychiatry. 2006;59(11):990-996. doi:10.1016/j.biopsych.2005.09.014.

21. Landis JR, Koch GG. The measurement of observer agreement for categorical data. Biometrics. 1977. 33:159-174.

22. Carter R, Lubinsky J. Rehabilitation Research-E-Book: Principles and Applications. Elsevier Health Sciences; 2015. 
23. Vanhoutte EK, Faber CG, Nes V, et al. Modifying the Medical Research Council grading system through Rasch analyses. Brain. 2012;135(5):1639-1649. doi:10.1093/brain/awr318.

24. Fan E, Ciesla ND, Truong AD, Bhoopathi V, Zeger SL, Needham DM. Inter-rater reliability of manual muscle strength testing in ICU survivors and simulated patients. Intensive Care Med. 2010;36(6):10381043. doi:10.1007/s00134-010-1796-6.

25. Reid WD, Dechman G. Considerations When Testing and Training the Respiratory Muscles. Phys Ther. 1995;75(11):971-982. doi:10.1093/ptj/75.11.971.

26. Myers BJ, lonta MK, Voss DE. Facilitación Neuromuscular Propioceptiva. Patrones y técnicas.; 2001. https://dialnet.unirioja.es/servlet/libro?codigo=302126. Accessed June 8, 2017.

27. Cutter NC, Kevorkian CG. Handbook of Manual Muscle Testing. 1 edition. New York: McGrawHill/Appleton \& Lange; 1999.

28. Jardim JR, Mayer AF, Camelier A. Músculos respiratorios y rehabilitación pulmonar en asmáticos. Arch Bronconeumol. 2002;38(4):181-188. doi:10.1016/S0300-2896(02)75186-7.

29. Soderberg GL. Kinesiology: Application to Pathological Motion. Williams \& Wilkins; 1997.

30. Wilson TA, Troyer AD. The two mechanisms of intercostal muscle action on the lung. J Appl Physiol. 2004;96(2):483-488. doi:10.1152/japplphysiol.00553.2003.

31. Lavietes MH, Grocela JA, Maniatis T, Fotulski F, Bitter AB, Sunderam G. Inspiratory Muscle Strength in Asthma. Chest. 1988;93(5):1043-1048. doi:10.1378/chest.93.5.1043.

32. Kinesioterapia. Ed. Médica Panamericana; 2000.

33. Brown PI, Johnson MA, Sharpe GR. Determinants of inspiratory muscle strength in healthy humans. Respir Physiol Neurobiol. 2014;196:50-55. doi:10.1016/j.resp.2014.02.014.

\section{Figures}




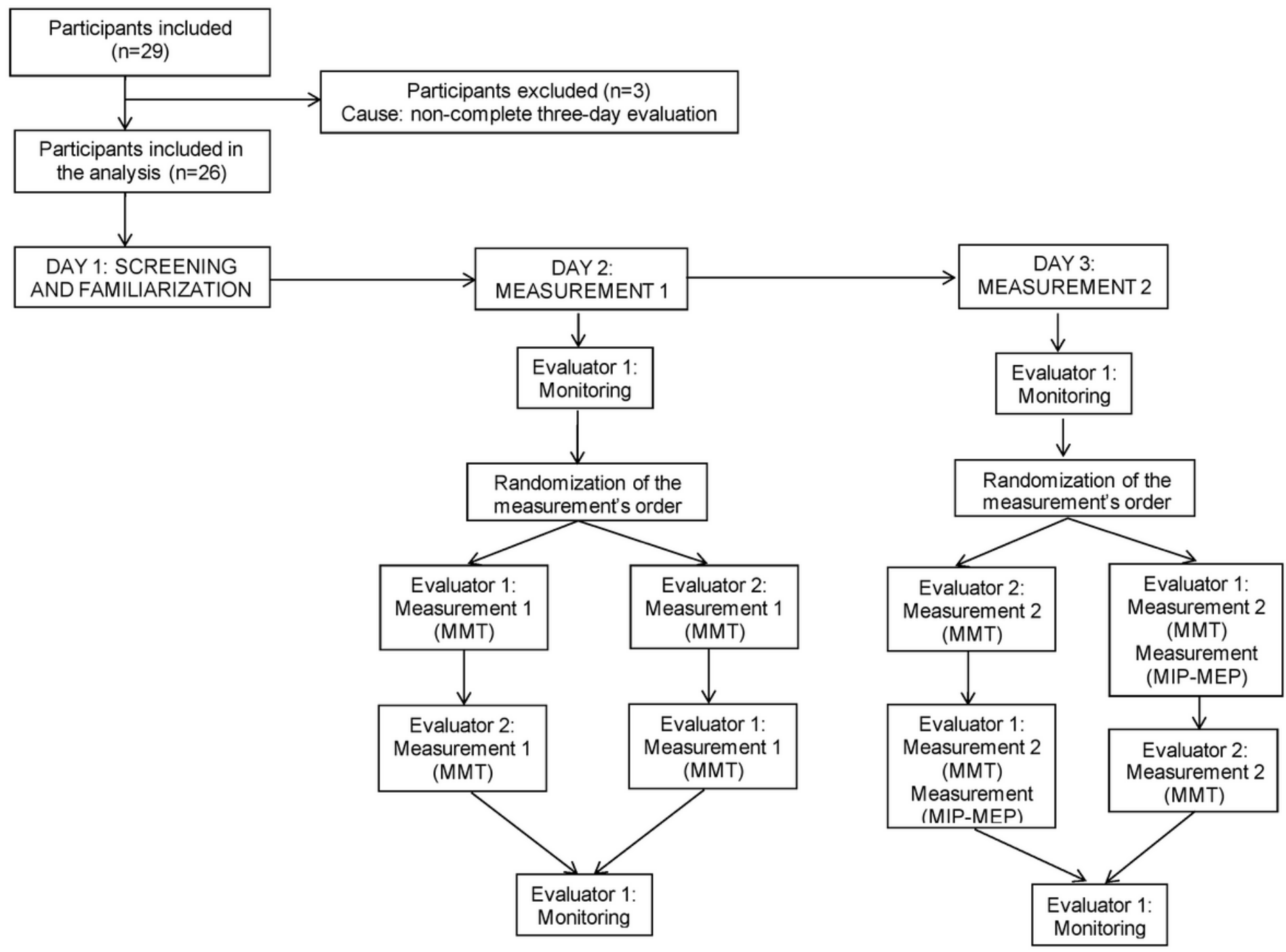

\section{Figure 1}

Flow - chart of study participants 


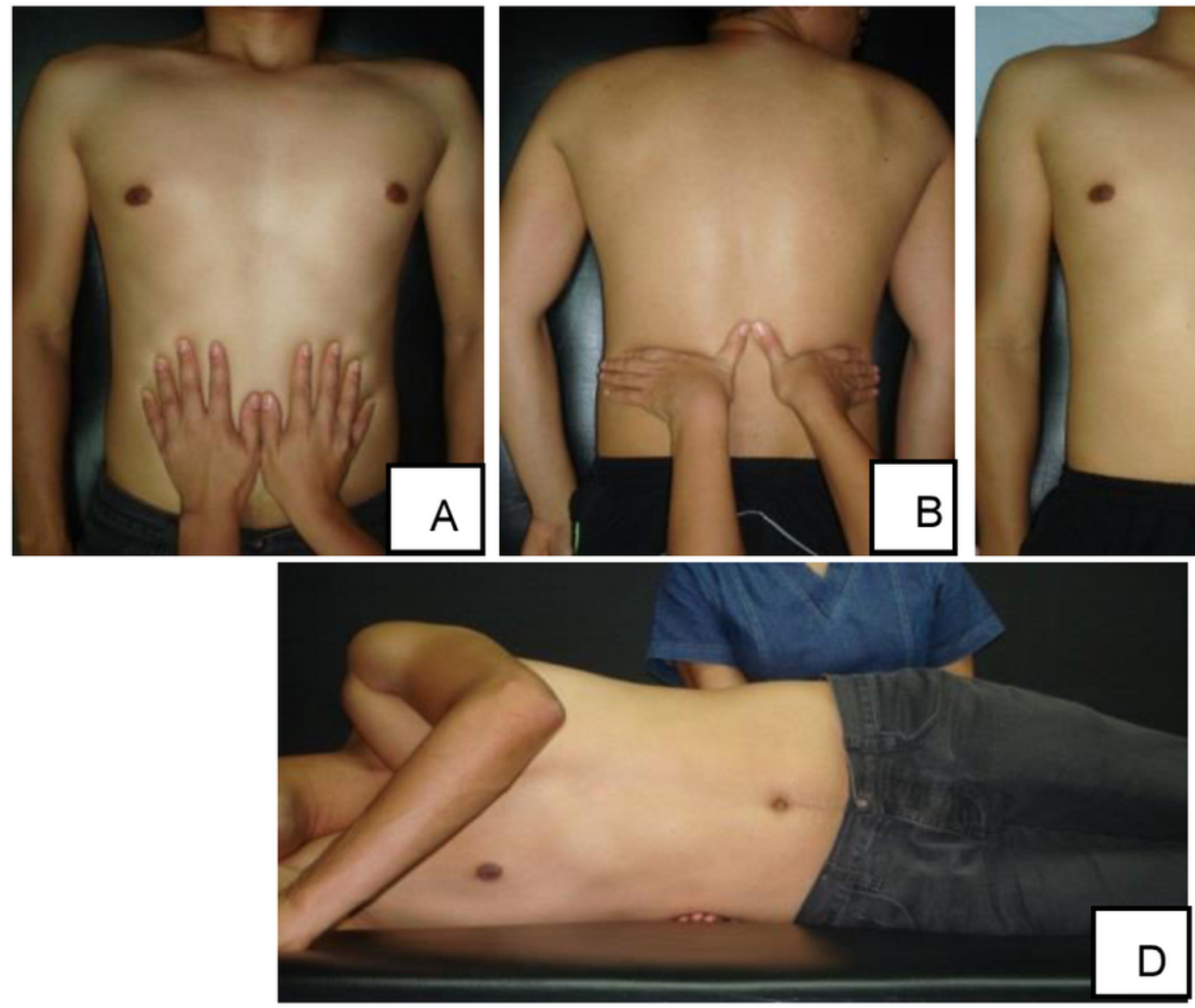

Figure 2

Evaluation of diaphragm muscle. A: Sternal fibers. B: Lumbar fibers. C: Palpation of weak fibers. D: Lateral fibers. 

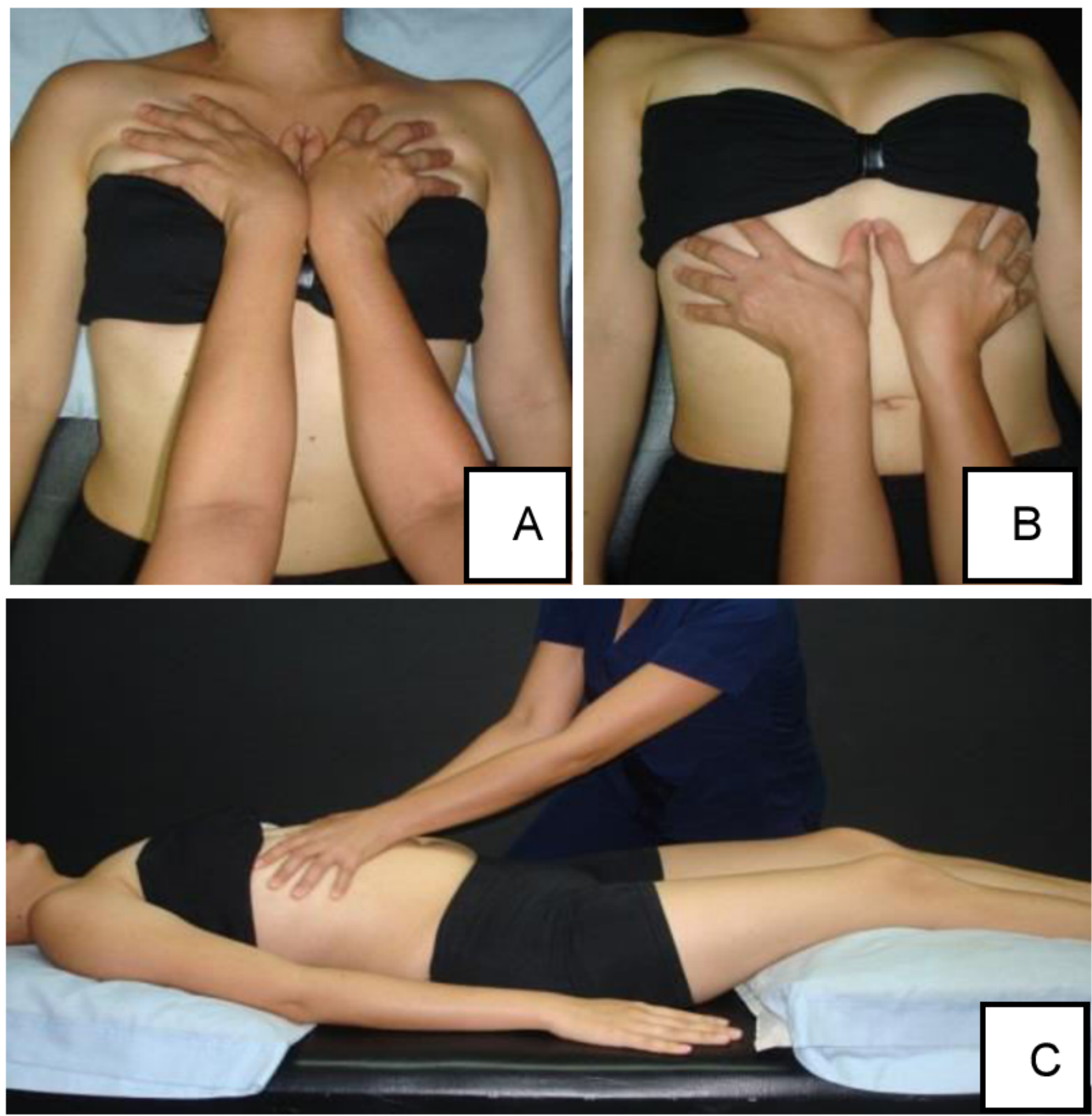

Figure 3

Evaluation of external intercostal muscles. A: Superior. B: Inferior. C: Posture for testing 

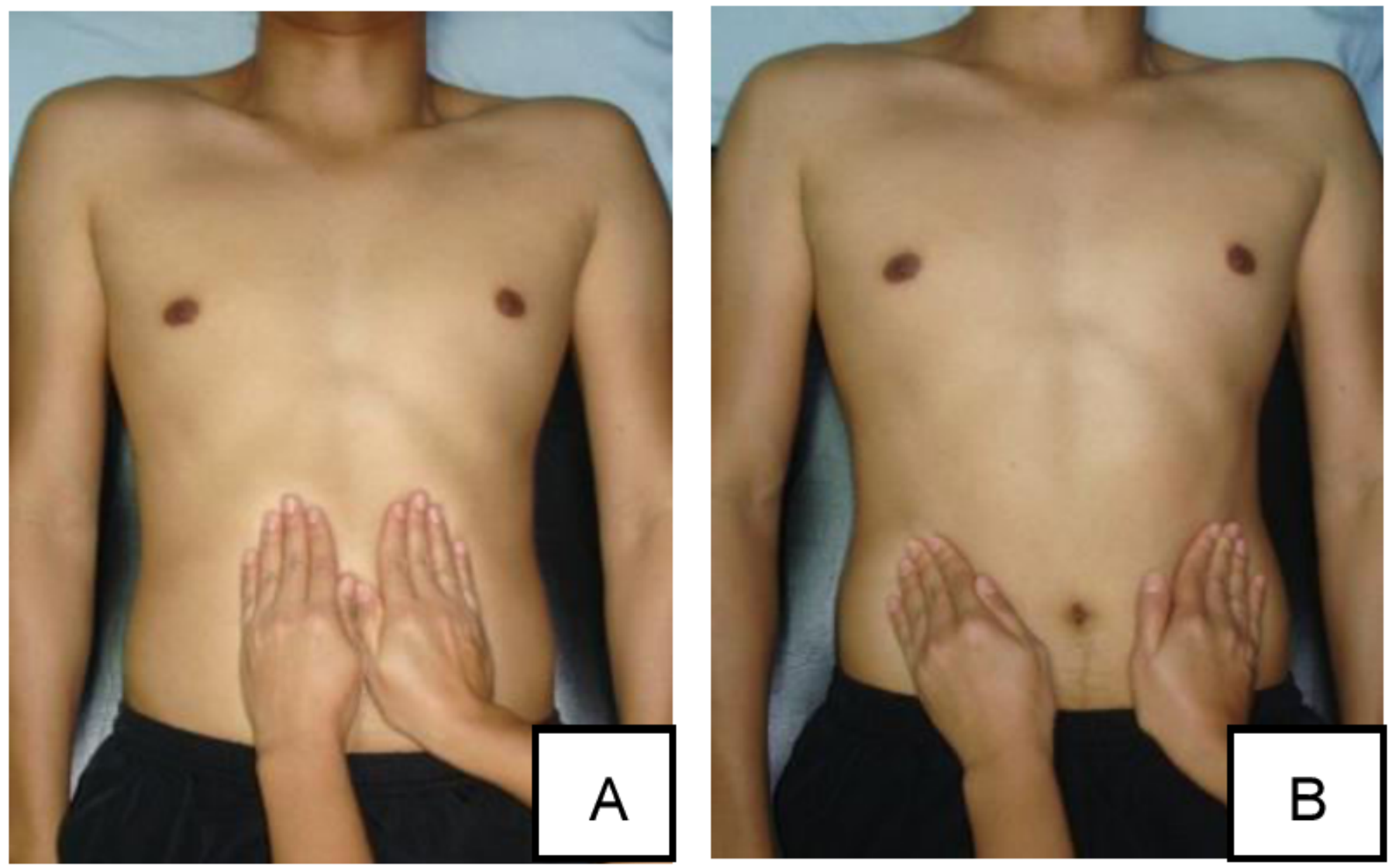

Figure 4

Evaluation of abdominal muscles. A: Rectus abdominis. B: Oblique muscles. 